\title{
Possible barriers for genetic counselors returning actionable genetic research results across state lines
}

\author{
Megan C. Roberts, $\mathrm{PhD}^{1}$, Elisabeth M. Wood, MS², Jill Bennett Gaieski, PhD, JD ${ }^{3}$ and \\ Angela R. Bradbury, $\mathrm{MD}^{2,4}$
}

With advances in genetic sequencing, researchers have greater opportunities to understand genetic contributions of disease to potentially improve clinical outcomes. At the same time, research teams must increasingly consider the return of clinically significant (i.e., actionable) genetic research findings to research participants. While at present there is no legal obligation for researchers to return incidentally identified research results from genetic sequencing, there is an emerging opinion that there may be an ethical duty to return genetic results obtained in the research setting that could impact a participant's clinical care. ${ }^{1}$ As such, researchers are now recommended to inform participants about the potential for incidental (not sought after, but incidentally identified) and secondary genetic findings (findings intentionally sought after) during the informed consent process, including how these results will be shared and honoring participants' right to decline results. ${ }^{2}$ Yet, how best to implement these emerging ethical obligations remains unknown. ${ }^{3}$

Given the limitations and complexity of genetic information, some have suggested that genetic counselors may be ideal brokers of informed consent and clinically actionable research findings. ${ }^{4}$ However, genetic counselor workforce shortages and professional costs of utilizing genetic providers (versus returning results by research staff, mail, or through participants' primary care providers) are potential barriers. ${ }^{4}$ Additional challenges exist for research cohorts that are not associated with clinical programs and for national or multistate cohorts, where providing in-person genetic counseling can be logistically prohibitive. One potential model to maintain the benefits of utilizing genetic providers is to provide remote genetic services (e.g., by phone or videoconferencing) to research participants through a single coordinating center. ${ }^{5}$

From a quality perspective, providing genetic counseling through a coordinating center may improve standardization of counseling and support return of results by experts in particular health conditions or by those who understand the research cohort's unique contextual factors. While face-toface counseling is the traditional clinical delivery model in genetic care, phone counseling has been used increasingly and has been found non-inferior to in-person counseling, at least for $B R C A 1 / 2$ counseling and disclosure. ${ }^{6,7}$ Further data are needed regarding outcomes for a broader range of genetic results and in the context of returning incidentally identified research results. Nonetheless, phone counseling is a practical approach that many are utilizing when face-to-face counseling is not feasible. ${ }^{4}$

Although the single coordinating center approach seems promising, requirements for genetic counseling licensure, which vary by state, are an underappreciated challenge. These requirements have been important to the genetic counseling profession, providing legitimacy to the field, facilitating genetic counseling reimbursement, and most importantly for the public's protection. ${ }^{8}$ Utah became the first state to license genetic counselors in 2002. Now, 18 states issue licenses; three are in the process of implementing new laws. ${ }^{8}$

Not only is there variation in whether states have licensure requirements, but also in how states define the scope of practice (e.g., some states have specific definitions, while others utilize professional definitions, such as the National Society of Genetic Counselors ${ }^{9}$ ), exemptions from licensure (e.g., students, those conducting research, those in occasional practice, or in rare disease cases), and the specific requirements for obtaining licensure (e.g., some require professional reference letters, fingerprints, and background checks). Importantly, genetic education and explanation of family history to help individuals understand genetic contributions of disease are considered within genetic counselors' scope of practice, regardless of any specific genetic testing/test result or even in the research context. This has implications for returning clinically actionable genetic research results by a genetic counselor through a coordinating center, as genetic counselors would need to adhere to licensure laws for each state where a participant resides, unless a research or other

${ }^{1}$ Division of Cancer Control and Population Sciences, National Cancer Institute, Rockville, Maryland, USA; ${ }^{2}$ Division of Hematology-Oncology, Department of Medicine, The University of Pennsylvania, Philadelphia, Pennsylvania, USA; ${ }^{3}$ Center for Advanced Medicine, Department of Medicine, The University of Pennsylvania, Philadelphia, Pennsylvania, USA; ${ }^{4}$ Department of Medical Ethics and Health Policy, The University of Pennsylvania, Philadelphia, Pennsylvania, USA. Correspondence: Angela R. Bradbury

(angela.bradbury@uphs.upenn.edu)

Submitted 20 August 2016; accepted 17 February 2017; advance online publication 20 April 2017. doi:10.1038/gim.2017.34 
applicable exemption applies. In our experience in the Penn Telegenetics Program, there are considerable costs associated with the legal assessment of state laws, which may change over time, and with obtaining and maintaining provider licensure. These costs may be prohibitive for some research teams and large national cohorts. Thus, major barriers exist in returning clinically actionable findings to research participants across state lines.

Licensure requirements are not unique to genetic counselors. Providers of remote or "telehealth" clinical services face similar challenges, as each state has its own professional licensure laws and practitioners are dependent on the scope of practice for the profession. ${ }^{10}$ While physicians generally need a license to practice medicine in all states, some states have research exceptions (e.g., for particular types of research, CA Section 2909(b) or a limited number of participants), or accept reciprocity (e.g., accept licensure from the state in which the physician resides) (personal communication, legal assessment for Penn Telegenetics Program, 2015-2016). Additionally, many states have specific telemedicine regulations, but vary in their definitions of telehealth and in which professionals are included in these regulations. For example, some accept a physician's state licensure for telehealth, as long as the physician registers with the state where they are providing services, while the majority require physicians to obtain licensure in the patient's state (e.g., Maryland COMAR 10.32.05.03) (personal communication, legal assessment for Penn Telegenetics Program, 20152016). Of note, in our program, we have found that most states do not specifically include genetic counselors in their telehealth regulations. Similarly, few include research or other exceptions for genetic counselor licensure (personal communication, legal assessment for Penn Telegenetics Program, 2015-2016). Thus, regardless of the provider (genetic counselor or physician), an assessment of the activities planned (i.e., how this aligns with professional scope of practice, legal licensure requirements, and any telemedicine regulations in the state where the participant resides) is necessary.

There are several options for overcoming these challenges, although each has potential limitations. First, genetic counselors partnering with a research team could obtain genetic counseling licensure from all requiring states where they anticipate disclosing results or counseling participants. However, we have found this solution costly in terms of the legal analysis and the time and resources necessary to obtain and maintain licensure in multiple states. For example, in addition to the initial application process, which varies by state in terms of complexity and financial costs, many states require the applicant to provide official verification of every state license the applicant already holds. For a genetic counselor already licensed in multiple states, this verification requirement greatly increases the time and resources needed to complete a single application. National licensure (similar to the Nurse Licensure Compact), permitting license reciprocity or research exceptions could be viable solutions. Defining professional standards for pretest and posttest counseling in both research and clinical settings could further address barriers, were they to be adopted uniformly in state licensure laws. Another strategy, which at least one for-profit company has used, is to hire a cohort of genetic counselors residing and licensed in different states. ${ }^{11}$ The limitation of this approach is that the counselors are not working within a coordinating center, and therefore may not achieve the collective contextual understanding of the participants' disease or research cohort. Another option is to return research results to participants' local health care provider, who would share the results and provide counseling and follow up. In addition to the above limitations, the potential downside of this approach is that local nongenetic providers may lack the expertise or resources to provide sufficient guidance, resulting in suboptimal patient outcomes. ${ }^{12}$ While primary care providers could obtain consultation from local genetic counselors, many patients and providers do not have access to genetic counselors or must travel long distances to meet with one. ${ }^{13}$

Perhaps the most practical and comprehensive alternative is web-based delivery of clinically actionable findings ${ }^{3}$ with local follow-up by genetic counselors. This strategy would likely avert licensure requirements while still providing a means to share initial results, and guidance on the importance of medical follow-up, and clinical confirmation testing, if indicated. ${ }^{14}$ Developed with genetic experts, Web-based interventions could account for the research cohort context and specific genetic findings, yet still be scalable. Such an approach would provide participants opportunities to learn at their own pace and revisit materials. ${ }^{14}$ Nonetheless, there would be costs associated with web development and followup care with local providers or genetic counselors, particularly if research results need to be confirmed. Thus, costs and limitations to each approach remain. While these costs could be included within a grant's research budget, this has the potential to reduce the available funds for the scientific aims and could negatively impact scientific progress given the limited funding environment. ${ }^{2,3}$ This commentary is not intended to be a comprehensive review of the issues. Defining practice standards for informed consent and disclosure, as well as other issues, will be important to consider moving forward.

While web-based return is an attractive scalable option, studies comparing the outcomes and costs of web-based alternatives to provider-mediated models are needed. In the interim, research teams should consider legal restrictions in using genetic providers for the return of actionable genetic research results in multistate studies, recognizing that these restrictions may change over time. If research teams are going to be responsible for providing clinically actionable genetic research results to participants, the field of genetic medicine must consider what additional licensure policies, infrastructure, and interventions will facilitate this new role and move the field forward to benefit the health of research participants and their families. 


\section{COMMENTARY}

\section{DISCLOSURE}

The authors declare no conflict of interest. At the time of this work, Megan Roberts was a Cancer Prevention postdoctoral fellow at the National Cancer Institute. The views and opinions expressed in this article are those of the authors and do not represent the views of the Department of Health and Human Services.

\section{REFERENCES}

1. Pike ER, Rothenberg $\mathrm{KH}$, Berkman BE. Finding fault? exploring legal duties to return incidental findings in genomic research. Georgetown Law J 2014;102:795-843.

2. Presidential Commission for the Study of Bioethical Issues. Anticipate and Communicate: Ethical Management of Incidental and Secondary Findings in the Clinical, Research, and Direct-to-Consumer Contexts. Washington, DC, 2013

3. Jarvik GP, Amendola LM, Berg JS, et al. Return of genomic results to research participants: the floor, the ceiling, and the choices in between. Am J Hum Genet 2014;94:818-826.

4. Forrest LE, Young MA. Clinically significant germline mutations in cancercausing genes identified through research studies should be offered to research participants by genetic counselors. I Clin Oncol 2016;34: 898-901.

5. Bradbury A, Patrick-Miller L, Harris D, et al. Utilizing remote real-time videoconferencing to expand access to cancer genetic services in community practices: a multicenter feasibility study. J Med Internet Res 2016;18:e23.
6. Schwartz MD, Valdimarsdottir $\mathrm{HB}$, Peshkin $\mathrm{BN}$, et al. Randomized noninferiority trial of telephone versus in-person genetic counseling for hereditary breast and ovarian cancer. J Clin Oncol 2014;32: 618-626.

7. Kinney AY, Steffen LE, Brumbach BH, et al. Randomized noninferiority trial of telephone delivery of $B R C A 1 / 2$ genetic counseling compared with in-person counseling: 1-year follow-up. J Clin Oncol 2016;34: 2914-2924.

8. Levenson D. Genetic counselor licensure proponents call for more states to adopt licensing laws: benefits of licensure extend to geneticists, counselors, and patients. Am J Med Genet A 2016;170A:8-9.

9. Resta R, Biesecker BB, Bennett RL, et al. for the National Society of Genetic Counselors' Definition Task Force. A new definition of genetic counseling: National Society of Genetic Counselors' Task Force report. J Genet Couns 2006;15:77-83.

10. Kocher R. Doctors Without State Borders: Practicing Across State Lines, 2014. [...]. HealthAffairsBlog. http://healthaffairs.org/blog/2014/02/18/ doctors-without-state-borders-practicing-across-state-lines/. Accessed November 29, 2016.

11. We are InformedDNA, 2016. InformedDNA Healthcare, Personalized. http:// www.informeddna.com/about-informeddna. Accessed November 29, 2016.

12. Plon SE, Cooper HP, Parks B, et al. Genetic testing and cancer risk management recommendations by physicians for at-risk relatives. Genet Med 2011:13:148-154.

13. Cohen SA, Huziak RC, Gustafson S, Grubs RE. Analysis of advantages, limitations, and barriers of genetic counseling service delivery models. J Genet Couns 2016;25:1010-1018.

14. Tabor HK, Jamal SM, Yu JH, et al. My46: a Web-based tool for self-guided management of genomic test results in research and clinical settings. Genet Med 2017;19:467-475. 